\title{
Ball Lightning as Source of High-Energy Particles When It Enters a Dense Medium
}

\author{
Alexander Oreshko ${ }^{*}$, Anna Oreshko \\ ${ }^{1}$ Departament of Physics, Moscow Aviation Institute (National Aerospace Research University), Moscow, Russia \\ ${ }^{2}$ National Research University of Electronic Technology (MIET), Moscow, Russia \\ Email: *A.G.Oreshko@gmail.com
}

How to cite this paper: Oreshko, A. and Oreshko, A. (2021) Ball Lightning as Source of High-Energy Particles When It Enters a Dense Medium. Journal of High Energy Physics, Gravitation and Cosmology, 7, 1484-1502.

https://doi.org/10.4236/jhepgc.2021.74091

Received: September 15, 2021

Accepted: October 22, 2021

Published: October 25, 2021

Copyright $\odot 2021$ by author(s) and Scientific Research Publishing Inc. This work is licensed under the Creative Commons Attribution International License (CC BY 4.0).

http://creativecommons.org/licenses/by/4.0/

\begin{abstract}
Experiments have been carried out to study the anomalous passage of laboratory-produced ball lightning through solid-state sheets. The passing of the ball lightning within the standard model can be explained by cascading generation of particles at entering of high-energetic protons of the ball lightning into a dense matter. The process of energy conversion of its own poloidal magnetic field of the ball lightning into the kinetic energy of its charged particles occurs in this case. The energy of protons becomes sufficient for the generation of charged pions and their subsequent cascade decay. The decay of pions leads to the appearance of negative and positive muons, as well as muon antineutrino and muon neutrino. This fact is confirmed by the presence of a passed ball lightning and a high potential of variable polarity in the region above the solid-state sheet after the ball lightning passing through it. The dark ball lightning also found was in the experiments. The laboratory ball lightning opens up new perspectives in many areas of research and applications and may have a positive impact on attempts to solve the energy problem based on muon-catalyzed nuclear fusion.
\end{abstract}

\section{Keywords}

Ball Lightning, Passage Through Sheets, Converse of Energy, Cascade Process, Muons, Neutrino, Muon-Catalyzed Fusion

\section{Introduction}

A number of processes and phenomena in Nature, including a ball lightning, have not got received a correct explanation until now [1]-[8]. The ball lightning as an extreme form of the matter existence is one of the strangest and least understood natural phenomenon, attracting the attention of researchers about two 
centuries [1]. Nobel Prize winner P.L. Kapitsa believed that "ball lightning is a small window into other world" [2]. It should be noted that ball lightning has the same ideal spherical form and the same structure as emitting stars [9] [10]. The results obtained by N.A. Kozyrev allowed him to make the assumption that radiation from stars that have a significant rotational moment propagates with a relativistic speed and has a high penetrating ability [11]. As is well known, the sources of radiation are also rapidly rotating pulsars, which periodically are sources of fluxes of fast particles. The hypothesis of the particles existence, that have mass, impulse and posses high penetration ability, was predicted many years ago by G-L. Le Sage [12]. The ball lightning has also significant rotational moment that it receives during its origin in crossed electric and magnetic fields [10].

The ball lightning there is a source of high density energy. Earlier observers found an abnormally high thermal yield when the ball lightning enters into the water tank under natural conditions. In these cases small sized ball lightning evaporates significant amounts of water. This fact is confirmed by a number of observers. The energy density of a ball lightning can be estimated by the consequences that it leaves after it affects an object. The existing estimations of the energy density the ball lightning show that this value is equal to $W_{i n}=10^{10} \mathrm{~J} / \mathrm{m}^{3}$ [5]. Observations have shown that ball lightning is able to penetrate not only through glass windows, but also through solid objects, including sheets of metal and plastic [13] [14]. The ball lightning is often observed in enclosed spaces, as well as in cockpits and aircraft fuselages, in which there are no high voltage sources and capacitors with a large electrical capacity, which are necessary to generate the ball lightning. The passage of ball lightning through solid objects and the appearance in closed rooms give sufficient reason to believe that ball lightning is a source of elementary particles. It is known that only neutrino has an extremely high penetrating ability. The appearance of the ball lightning inside of an airplane is dangerous because it can cause a short circuit in a computerized system of control and, therefore, lead to a crash. A feature of the process of interaction of the ball lightning with the surface of a solid is the absence of imprints of charged particles on the surface of a body through which the ball lightning passes. Several attempts have been made to explain the high penetrating ability of the ball lightning. To explain the phenomenon of the penetration of ball lightning through glass, model has been proposed [15]. According to model [15] a ball lightning can appear inside a house or a plane cockpit during a thunderstorm on a closed glass window. This can occur due to accumulation of atmospheric ions on the isolating surface of the glass (or plexiglass) window. The collected surface charge can produce electric fields inside the cockpit or a room which are sufficient to sustain an electric discharge that creates a ball lightning according to [15]. However, there is no sufficient reason to believe that the electric field strength inside the cabin will be sufficient to generate the passed ball lightning. 
In [16], a model was proposed, according to which the atmospheric ball lightning makes a hole of very small diameter in the glass and penetrates through the glass without breaking it. This model explains the process of passing the ball lightning as a result of its mass transfer through a small hole from one side of the window glass to the other [16]. However, this can only be the case when the surface tension force dominates over the forces that are determined by the presence of electric and magnetic fields. In fact, the proposed model of the passing process does not take into account the presence of charges inside the ball lightning. This model also contradicts the high-energy ball lightning model [4] [6] [17] [18]. It should be noted that in a number of experiments carried out in [10] [14] [18] with a laboratory produced ball lightning the holes in glass were not observed. The models [15] [16] are valid only for explaining the passage of the ball lightning through dielectric materials. All currently existing models of ball lightning do not allow explaining the process of their penetration through sheets that are made of metal. The assumption that there is a particle that can pass through solids without any noticeable effect-a neutrino, which in the presence of an excited nucleus can take on a lower energy level than is required for the formation of pairs, and generate a stable state in the form of a "heavy neutrino" is given in [19].

The phenomenon of anomalous passing the radiation through solid body targets is also observed in laser physic - a fluxes of fast particles (including relativistic protons, electrons, and positrons) and a magnetic field from the back side of the target were registered in a number of experiments at intense interaction of laser radiation with the target [20] [21]. The phenomenon of the anomalous ball lightning penetration or super-passability was detected in the experiments [14] [22] is in some sense similar to the known phenomenon of helium super-fluidity, as noted in [10]. But in this case entirely different physical processes occur. The passing of the ball lightning through a solid body is one of the unsolved physical problems.

It should be noted that linear and ring accelerators are used in high-energy physics and technology to obtain elementary particles. The accelerators of such type consist of a proton source and a large number of accelerating modules. The disadvantage of existing installations is not only their very long length-they also require a high vacuum and significant consumption of electrical energy. The outstanding naturalist F. Bacon noted many years ago that all the best is in Nature and should be used for people. It should be noted that physical processes in Nature are implemented in a highly rational way and phenomena are observed that give sufficient grounds to believe that there are natural sources of elementary particles. The principle of operation of such sources differs significantly from the principle used in linear and annular accelerators.

The main goal of this paper conclude in creating the theoretical model that qualitatively describes the experimental results obtained by the authors on the anomalous penetration of the ball lightning through a dense matter, including 
solid objects. Studies of the ball lightning give an opportunity to obtain a principally new knowledge in physics. The time to open "a small window into a other world" has come in accordance to [2].

\section{Experimental Results on Passage of the Ball Lightning through the Solid-Body Sheets}

In experiments on studying the passage of the ball lightning through glass, plastic and metals, the ball lightning obtained in the laboratory, which is quasi-neutral in whole, was used. It consists of a core with excess negative charge and an outer spherical shell with excess positive charge. The distance between the core and the shell exceeds the Debye screening length. The generation of ball lightning in plasma occurs as a result of charge separation using crossed fields due to the fact that the external force due to the electric field exceeds the force of the Coulomb interaction between electrons and plasma ions. The mass of the experimental setup with a high-voltage transformer and a start-up unit is approximately 1500 $\mathrm{kg}$. The experiments were carried out at normal atmospheric conditions on the experimental facility "Prometheus" that was equipped a device for obtaining of the ball lightning-an electric-discharge spheretron [10]. Figure 1(a) shows a scheme of experiments for studying of the ball lightning. A description of experimental installations for producing the ball lightning is given in [10] [17] [18] [22]. An optical image of ball lightning $50 \mathrm{~cm}$ in diameter in the absence of a solid-state sheets is given in Figure 1(b). The registration of the optical images of

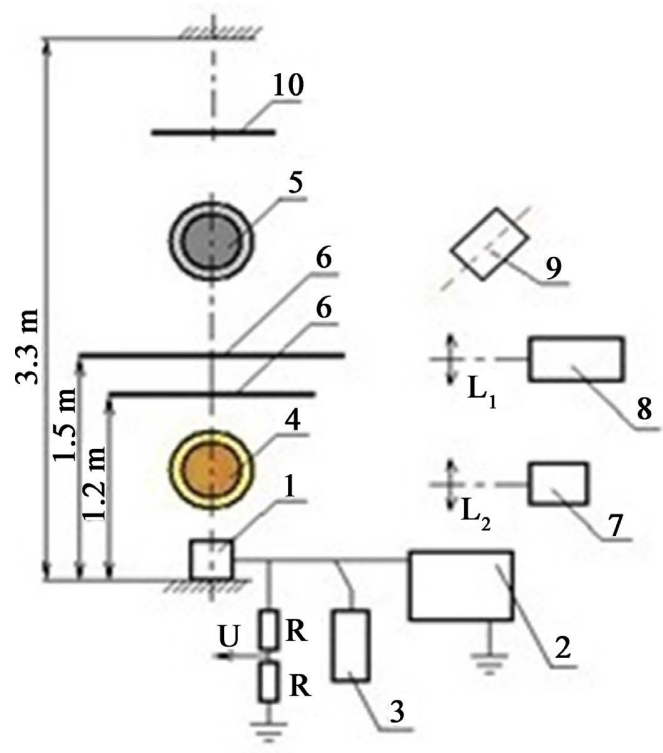

(a)

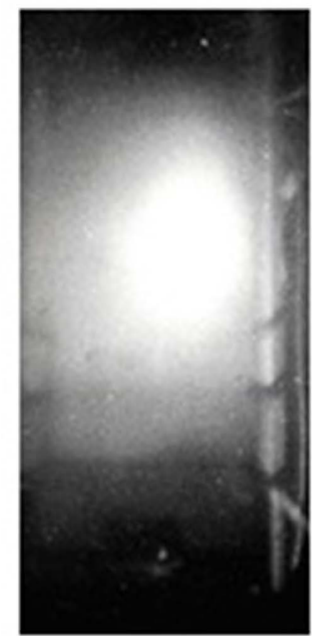

(b)

Figure 1. (a) A scheme of experiments. Designations: 1-spheretron; 2-capacitive energy storage; 3-starter unit; 4, 5-ball lightning before and after passage of a solid-state sheets; 6-a solid-state sheet; 7-electron-optical converter EP-15; 8-electron-optical camera FastCam-5; 9-neutron detector; 10-probe (or collector); L-lense. (b) An optical image of ball lightning $50 \mathrm{~cm}$ in diameter in the absence of the solid body sheets. 
ball lightning by its luminosity was carried out by means of the electron-optical converter EP-15 with resolution in space and time and electron-optical camera FastCam-5.

The maximum sensitivity of converter EP-15 was in the yellow-green range of spectrum. The facility is equipped with a plexiglass chamber used in the experiments on the ball lightning penetration through thick solid body objects. Figure 2 (a) shows general view of the experimental region with a plexiglass chamber on the "Prometheus" installation.

The plexiglass chamber was used only in experiments with very heavy absorbing elements, which consisted of lead and lead blocks. At the top of the camera were installed solid-state sheets, which were previously called absorbing filters. The term "absorbing filter" meant a solid body that partially or completely absorbed flows of charged particles and electromagnetic radiation. However, this term is not correct because there is no absorption of the ball lightning when it passes through solid-state sheets. The angle between the direction of entrance of the ball lightning and the plane of the solid-state sheets was equal to 90 degrees. Glass, aluminum and plexiglass sheets were installed on shelves that were made on the side surface of vertically installed gypsum boards-see Figure 3(b). The solid-state sheets were not grounded.

First we investigated the penetration of the ball lightning through glass sheets. Then we used plexiglass, aluminum and steel sheets as solid-state objects. A lead objects, which consisted of a sheet and blocks with a total thickness of $6 \mathrm{~cm}$, were placed in the upper part of the chamber (see Figure 2(b)), since, due to the large mass of lead blocks, it was impossible to install them using the side shelves.

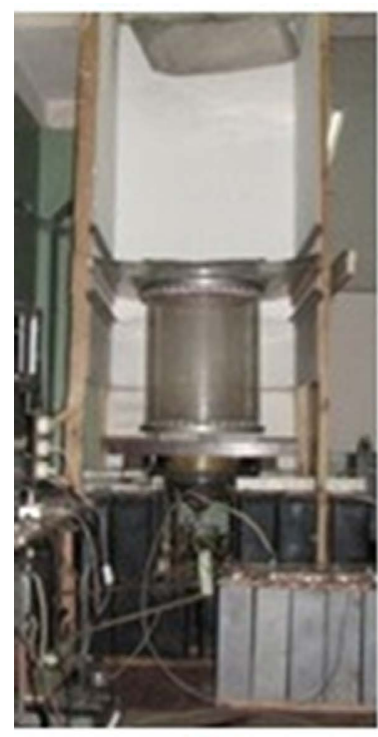

(a)

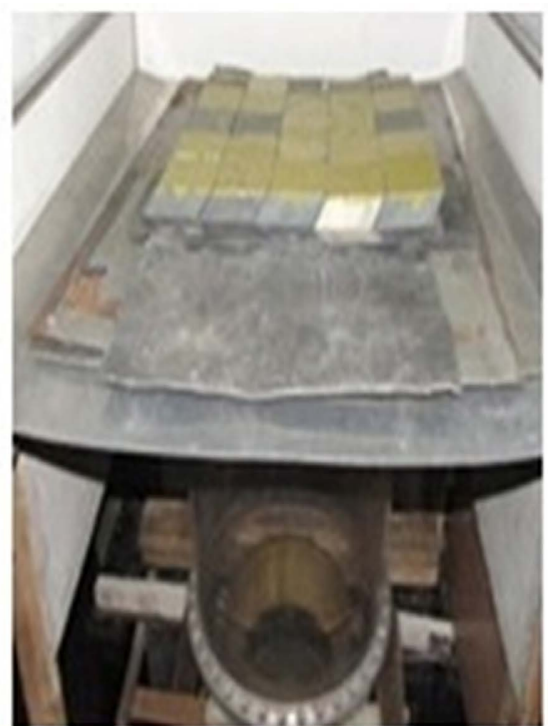

(b)

Figure 2. (a) General view of the "Prometheus" experimental facility with plexiglass chamber, which was used in experiments of passage of the ball lightning through solid body objects. (b) The solid-state object consisting of sheets aluminum, steel, lead sheet of thickness $1 \mathrm{~cm}$ and $5 \mathrm{~cm}$ lead blocs BS-50 on the top of the plaxiglass chamber. 
The use of a wide range of solid-state materials in experiments was due to the intention to confirm the assumption that ball lightning is created outside the aircraft and only then penetrates into its fuselage. Unlike submarines, aircrafts do not have powerful enough electric sources, in order to create the ball lightning. The length and width of solid-body sheets were respectively equal to $100 \mathrm{~cm}$ and $94 \mathrm{~cm}$. A box was made from three gypsum plasterboards in order to exclude possible effect of reflected and re-reflected plasma luminosity (created in the discharge) during the registration of optical images. The width of the plasterboard box was $94 \mathrm{~cm}$ and the height was approximately $2.5 \mathrm{~m}$. An image of the ball lightning between two a glass sheets is given in Figure 3(a). Figure 3(b) shown the ball lightning an ellipsoidal shape that has passed through two plexiglass sheets thickness of $4 \mathrm{~mm}$ every. The image of the ball lightning that has passed through $1.5 \mathrm{~mm}$ aluminum sheet and is entering into a $4 \mathrm{~mm}$ plexiglass sheet is given in Figure 3(c). The images in Figure 1(b) and Figure 3(a) were obtained with an EP-15 electron-optical converter. In Figure 3(a), we can see the fluxes of particles, which are directed through the upper sheet in the direction of motion of ball lightning. This means that there is generation of fast particles from the ball lightning in the direction of its motion. The sensitivity of the photographic film which is used for the optical registration in the electron-optical converter was equal to 3200 units. The images of the ball lightning in Figure 1(b) and Figure 3(a) were received with an exposure of 5 ns. In Figure 3(a) there is also an optical distortion due to reflection and re-reflection of the light between two glass sheets. The difference in the diameters of the ball lightnings in Figure 1(b) and Figure 3(a) is associated with the difference of energy values in the used capacitive energy storages of the experimental facility "Prometheus". The images in Figure 3(b) and Figure 3(c) was obtained by a high-speed camera FastCam-5. It was not possible to get series of good quality images of the ball

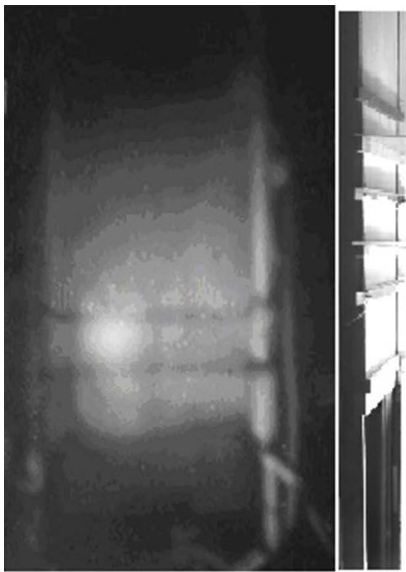

(a)

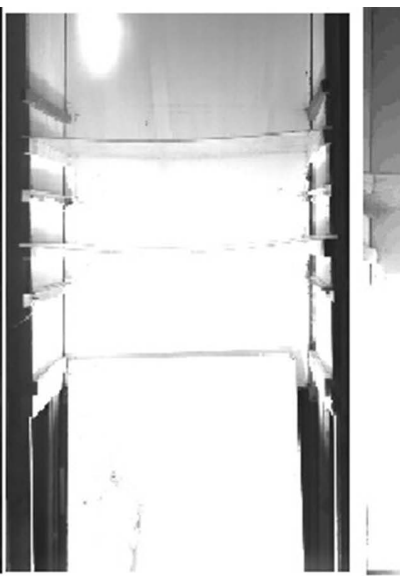

(b)

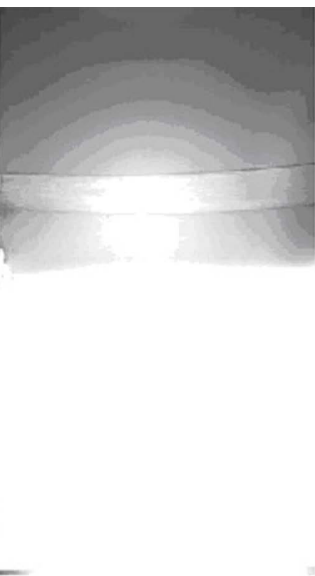

(c)

Figure 3. (a) The image of the ball lightning between two a glass sheets. (b) The image of the ball lightning an ellipsoidal shape that has passed through two plexiglass sheets. (c) The image of the ball lightning that has passed through $1.5 \mathrm{~mm}$ aluminum sheet and is entering into a $4 \mathrm{~mm}$ plexiglass sheet. 
lightning using a black-and-white camera. This requires a camera with an electron-optical converter with the maximum of sensitivity in the blue part of the spectrum.

Visual observations of the ball lightning were carried out using a blue optical filter because the brightness of the plasma glow in the discharge zone was always higher than the brightness of the resulting ball lightning and due to the possibility of damage to the eyes. A good visibility of ball lightning was in the zone of its generation, as well as in the area of obstacles such as the solid-state sheets or the ceiling of the laboratory, which were encountered on the way of its movement upward at great speed. The electric lights in the laboratory were turned off during the experiments. However, at during the start-up of the experimental setup, there was always intense background radiation due to the glow of the plasma in the discharge. An illustration with results of visual observations of the ball lightning passing through the solid-state sheets is given in Figure 4. In the experiments it was revealed that when the ball lightning passes through the thin solid-state sheet, the luminosity maximum is located in the region approximately corresponding to the diameter of the outer spherical shell-see Figure 4(d). With increasing of the thickness of the solid-state sheet the luminosity intensity is reduced and at some thickness value only an aura is visible in the area above the solid-state sheet (see Figure 4(e)). With further increase of the thickness of the solid-state sheet during the experiments only a black spherical formation (a black ball lightning) has been observed above the sheet [10] — see Figure 4(f). The diameter of the black ball lightning becomes approximately equal to the diameter of core of the ball lightning which approaches to the solid-state sheet. An analogy between the ball lightning and a small a black hole was presented in [23]. As good known, the radiation intensity of the black hole is not sufficiently for its optical registered. It should be noted that black color of the ball lightning can't be a base for an analogy between the ball lightning and the black hole. The ball lightning has its own specific features. It is impossible to carry out an optical recording of the black ball lightning due to the lack of radiation, but, contrary to the black hole, the black ball lightning is possible to observe visually at during

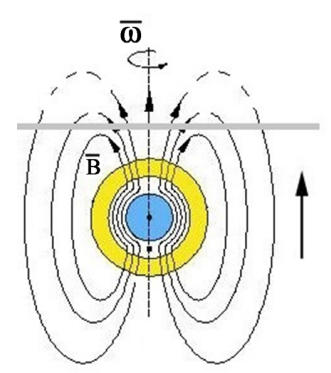

(c)

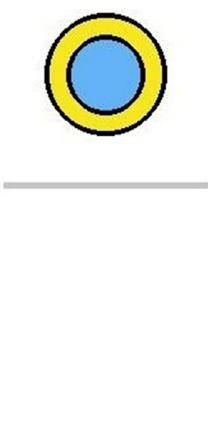

(d)

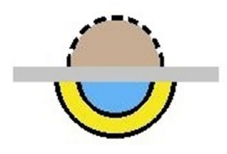

(e)

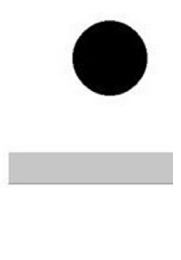

(f)

Figure 4. Illustrations of: (c) a ball lightning entering into thin a solid-state sheet; (d) a ball lightning that has passed through the thin solid-state sheet; (e) an aura on top of the solid-state sheet; (f) dark ball lightning that has passed through the thick solid-state sheet. 
experiments. The vertical (axial) speed of the ball lightning in the zone of its generation exceeds $5 \times 10^{8} \mathrm{~cm} / \mathrm{s}$. High speed of movement is achieved due to the acceleration of the ball lightning particles in crossed alternating electric and magnetic fields, with help of which structural elements of the ball lightning are formed as a result of charges separation. The vertical speed of ball lightning in the generation zone differs greatly from the speed after its transition to a drift state in space [7].

It should be noted that black ball lightnings were also observed in experiments in absence of the solid-state sheets at a very low charge in the capacitive storage ( $Q=0.25 \mathrm{C}$ ). The black ball lightnings obtained in the experiments with a small charge pass through the solid-state sheet (if it present) as well as the conventional ball lightnings, which were obtained with a charge in the capacitive energy storage equal to $Q=1-3 \mathrm{C}$. The existence of the black ball lightning is associated with a small value of its charge.

\section{Electric and Magnetic Fields of the Ball Lightning}

Earlier it was obtained that ball lightning consists of a core with surplus negative charge and an external spherical shell with surplus positive charge [9] [10]. An optical images of the ball lightning obtained on the "Prometheus" facility in the absence of light in the laboratory, the schemes of its structure and orientation of fields are given accordingly in Figure 5(a), Figure 5(b) and Figure 5(c). The distance between the core and the outer shell is greater than the Debye screening length. The ball lightning is an electrical domain of spherical shape which rotates with big angular velocity $\omega$ about an axis of rotation. This confirms the fact that ball lightning is a spherical electrical domain. A strong electric field is always exists between the elements of the electrical domain [24]. The electric field of the ball lightning has both radial- $\boldsymbol{E}_{r}$ and ambipolar components- $\boldsymbol{E}_{\text {amb }}$ (see Figure 5(c)).

The ambipolar electric field there is between the core and the outer spherical

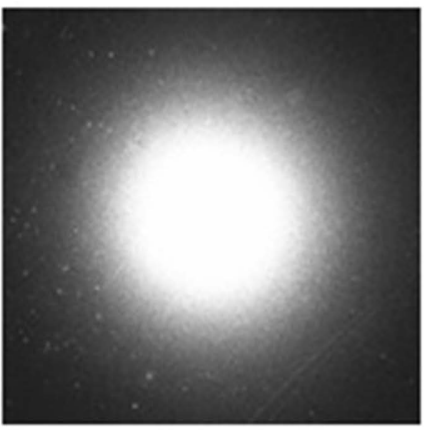

(a)

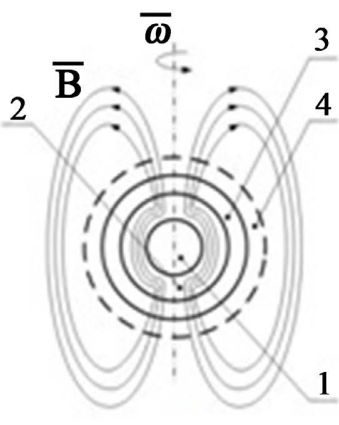

(b)

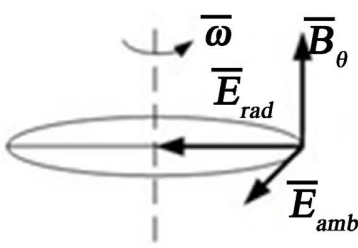

(c)

Figure 5. (a) An optical image of the ball lightning obtained on the "Prometheus" facility in the absence of light in the lab. (b) A scheme of ball lightning structure. Designation: 1-core; 2 -intermediate layer; 3-outer spherical shell; 4-halo; $\boldsymbol{B}$-lines of induction of the poloidal magnetic field; $\boldsymbol{\omega}$-angular rotation velocity. (c) A scheme of fields orientation in equatorial plane between an elements of the ball lightning. 
shell. This field exists due to the fact that the rotating outer spherical shell causes a rotation of the core, which is caused by electrostatic interaction between the elements with surplus positive and surplus negative charges. The experiments have shown that the ball lightning has a poloidal magnetic field- $\boldsymbol{B}_{\theta}$ [10] [22]. The ball lightning also has a considerable rotational moment, which it acquires in the plasma vortex during its generation [25]. The rotating outer spherical shell, i.e. closed ring current of charged particles-protons, creates a poloidal magnetic field-the magnetic dynamo. There is also a halo outside the outer spherical shell. The halo existence is associated with the outer electric field between the outer spherical shell and the atmosphere surrounding of the ball lightning.

An analysis of distribution of the glow intensity of the laboratory ball lightning (see Figure 6) used in these experiments and the ball lightnings recorded by observers during a thunderstorm in nature (see Figure 7) using the Scion Image program (created by the Scion Corporation) and Abel's inversion shows that the glow intensity of the core is significantly less than the glow
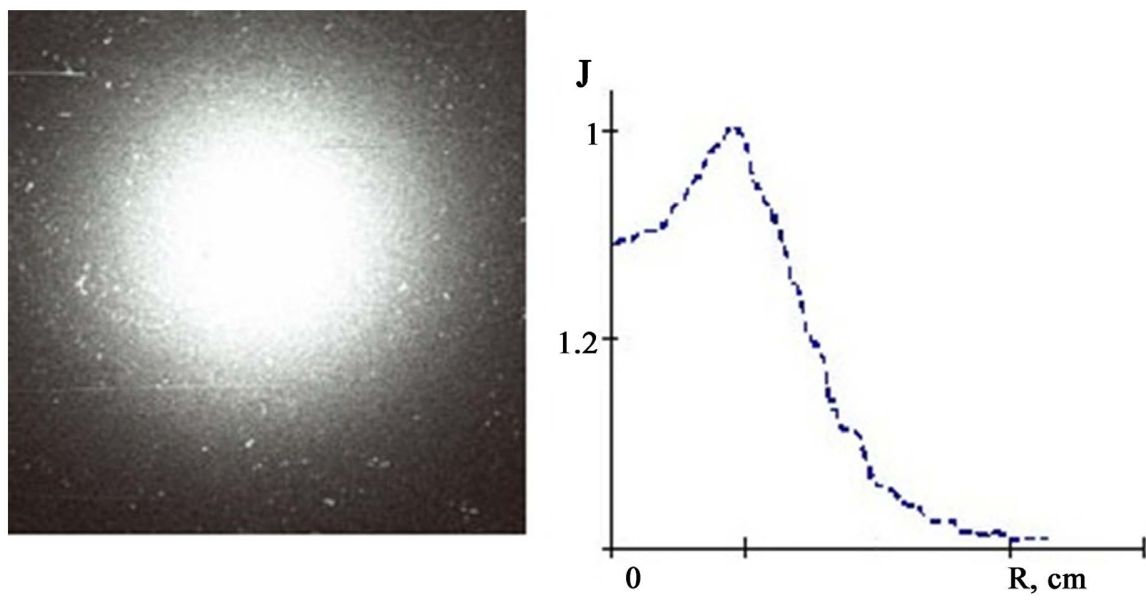

core:shell:halo

Figure 6. Radial distribution of glow intensity for the ball lightning used in the experiments.
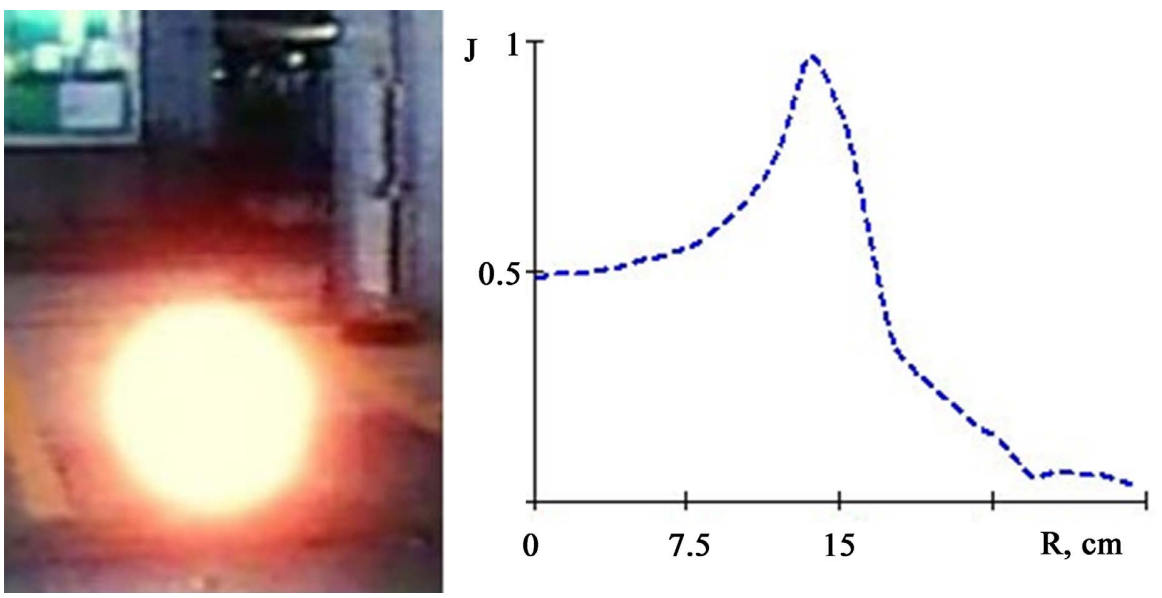

Figure 7. Radial distribution of glow intensity for the natural ball lightning. 
intensity of the outer shell.

The intensity of the outer electric field is significantly lower than the intensity of the radial electric field between the elements of the ball lightning. The resulting value of the electric field intensity is equal to the vector sum of all components. In process of the ball lightning generation the intensity of the electric field is changes. Experiments have shown that the ball lightning has a strong electric field [10] [17]. The radial electric field strength considerable times exceeds the limit value for electrical breakdown in an air. Electrical breakdown between the core and the outer shell of the ball lightning does not occur due to the presence of a magnetic field, which prevents the movement of electrons in the radial direction and due to the absence of particles (conductive matter) between its the structural elements.

An experiments were carried out in which bursts neutrons were registered during generation of ball lightning, which exceed the level of natural neutron background [19]. The value of the ball lightning potential, which exceeds the value required for the nuclear photoeffect, was also measured. These experiments made it possible to create a relativistic proton-electron model of the ball lightning. The ball lightning core consists mainly of electrons and an external spherical shell consists mainly of protons [19]. It should be noted that the orientation of intensity vectors of electric and magnetic fields in a ball lightning is similar to that of such field vectors in the particle accelerator of cyclotron type. The presence of strong crossed electric and magnetic fields makes it possible to consider ball lightning as an autonomous charged particle accelerator "built" into the atmosphere, as noted in [10]. As it is known, the maximum energies of charged particles in cyclotrons are approximately $20-25 \mathrm{MeV}$. This value is significantly higher than the value that was obtained earlier by the author using the method of absorbing [10].

The long existence of ball lightning is due to the fact that its rotating structural elements consist of a core and an external spherical shell with surplus space charges [10]. The excess charge of one sort and the lack of charge of other sort in the core or in the external spherical shell significantly reduce the possibility of an electron capture by the nearest ion, therefore the recombination processes in the ball lightning become impossible. As is good known, laboratory plasma is a locally quasi-neutral form of the state of matter in accordance with the classical definition of the Nobel Prize laureate I. Lang-muir. The fact that ball lightning is a quasi-neutral object as a whole, i.e. because of its structure, gives reason to believe that such a state can be interpreted as the fifth form of the existence of matter in Nature. The ball lightning core is located in the area with the minimum of induction of the poloidal magnetic field. The magnetic field is created by a closed circular current of relativistic particles that are present in the outer spherical shell. It should be noted that plasmoids with a high penetrating ability were found in a high-current vortex discharge during the generation of the ball lightning [25]. 


\section{Energy Conversion at Entry the Ball Lightning into a Dense Medium}

The exceptionally high penetrating power of the ball lightning gives enough reason to suggest that at interaction of the ball lightning with the dense matter take place a generates neutrino [19] [26]. An attempt to explain the phenomenon of the passage of ball lightning on the basis of the cascade process, which takes place in extensive air shower was undertaken in [26]. As known, the extensive air showers occur when the high-energetic particles enter a dense atmospheric layers. For the first time this phenomenon has been discovered in [27]. During the extensive air showers a cascade process of interaction between primary cosmic rays and atmosphere occurs. This interaction creates the secondary components of cosmic rays, namely, elementary particles. As you know, the primary high-energy particles are protons. The external shell of the ball lightning consists of protons [17]. The difference between the process of interaction of the cosmic rays with the atmosphere and the process of the passing of the ball lightning through the solid-state object is following. First of all, the ball lightning interacts with a matter which density many times higher than the density of air, and, secondly, the energy of the primary particles is lower than the energy such particles in extensive air showers. It should also be noted that the entrance of the ball lightning into the solid-state sheet occurs in the presence of its own poloidal magnetic field, which must enter the solid-state sheet having a finite magnetic permeability (see Figure $4(\mathrm{c})$ ). The magnetic field which is created by the ring current of the particles in the outer shell of the ball lightning is much higher than the magnetic field of the Earth. The difference between the entrance of ball lightning into the solid-state sheet and the entrance of high-energy protons in an extensive air shower is also that the spins of the ball lightning protons have the same orientation-therefore, the ball lightning is a spin-polarized object. It is obvious also that the magnetic field cannot penetrate through thick solid-state sheet such as lead $6 \mathrm{~cm}$ thick. If across the direction of motion of the black spherical formation is installed a sheet of metal so that it has rotational and vibrational degrees of freedom, i.e. hang it in one point, then the interaction of the black ball lightning with a sheet leads to oscillation of this sheet. This means that the particles from which consist the black ball lightning possess the impulse, i.e. they has not only speed but also mass. The potential measurements by means of the probe under the ceiling indicate that the signals have a variable polarity. This means that the ball lightning that has passed through the solid-state sheet consists of positive and negative charges. The generation of muons and neutrino during the interaction of the ball lightning with thick solid-state plates is confirmed by the presence of the black ball lightning, which passed through a sheet and blocks of lead with a total thickness of $6 \mathrm{~cm}$ (see Figure 2(b)).

The presence of the nuclear photoelectric effect during the generation of ball lightning and the measurement of its potential showed that the energy of protons in the outer shell of ball lightning is $20 \mathrm{MeV}$ [17]. This energy value is in- 
sufficient to generate pions and kaons. In the first approximation, we can assume that the stability of ball lightning is achieved when the centrifugal force is balanced by the force between the structural elements of the ball lightning caused by electric field. The equation of force balance for ball lightning can be written in the form

$$
\frac{m v^{2}}{R}=q \boldsymbol{E},
$$

in which $m$ is the mass of the proton, $v$ is its velocity, $R$ is the radius of the ball lightning; $q$ is its charge; $\boldsymbol{E}$ is the strength of the electric field inside the ball lightning. Taking into account the radius $(R=12.5 \mathrm{~cm})$ and electric field strength ( $E=2.3 \mp 10^{4} \mathrm{~V} / \mathrm{cm}$ ) obtained in [10] it's possible to find that the energy of protons in the external shell of ball lightning does not differ at best from the energy obtained during the generation of ball lightning in a cyclotron-type source. However, this value of proton energy is insufficient to realize a cascade process at the entrance of ball lightning into a dense matter. The phenomenon of anomalous passage of ball lightning through metal absorbers had observed by authors several thousand times in the experiments since 2006 year. The absence of a theory that can give a correct explanation for the observed phenomenon is not a reason for negating the experiment. This means that protons gain enough energy to generation pions at time when they enter a dense matter. This process is similar to the process of cosmic rays acceleration that enters the gas clouds in the interstellar or intergalactic matter in magnetic field [28]. The entrance of a spherical ball lightning into a dense matter is equivalent to the process of expanding the sunspot. The process of expanding the sunspot as well as the process of entering the ball lightning into a dense matter with final permittivity is accompanied by a change of the magnetic field. As known the acceleration of charged particles occurs in time-dependent magnetic fields. The generation or the disappearance of the magnetic field is accompanied by the appearance of an induced electric field in which electrons and protons can be accelerated up to relativistic values of energy [28]. The process of obtaining energy by protons during the interaction of ball lightning with a dense matter can be explained elementary by means of a cyclotron mechanism [28]. The magnetic flux $\Phi$ variation in time creates a potential $U$ in accordance with $\mathrm{M}$. Faraday's law

$$
-\frac{\mathrm{d} \Phi}{\mathrm{d} t}=U
$$

The magnetic flux at first approximation is given by expression

$$
\Phi=\int \boldsymbol{B} \cdot \mathrm{d} S=B \cdot \pi \cdot R^{2} .
$$

The value of energy that the charged particle gains at the change of magnetic field with induction $\boldsymbol{B}$ can be found by the formula [28]

$$
\boldsymbol{E}=e U=e \pi R^{2} \frac{\mathrm{d} \boldsymbol{B}}{\mathrm{d} t} .
$$

Charged particles of the ball lightning with a radius of $0.1 \mathrm{~m}$ with magnetic 
field induction $\boldsymbol{B}$ in a few nanoseconds of interaction of the ball lightning with a solid-state sheet, increase the energy from 20 to $140 \mathrm{MeV}$. To calculate the increase of particle energy, it is necessary to have accurate values of the magnetic field induction and the time of its change. It is impossible to experimentally obtain exact values of these values because ball lightning is an object with strong own fields. However, it is impossible to refute the fact that when ball lightning interacts with a dense matter, the process of converting the energy of the own magnetic field of the ball lightning into the kinetic energy of its charged particles occurs. Obvious evidence of this phenomenon is visually observed ball lightning passing through solid-body sheets, and it also consists of electrical charges similar to incoming ball lightning. The energy gained by protons when ball lightning at enter into a solid-body sheet becomes sufficient for the formation of pions and the implementation of a cascade process of generation of elementary particles.

It is also possible that when the ball lightning enters a dense matter between its core and outer shell, an electrical breakdown will occur, which will lead to the generation of an intense transverse electromagnetic wave diverging in a plane perpendicular to the ball lightning axis. A similar phenomenon takes place in emitting stars and it realized when the balance of the acting forces between the elements of the star is disturbed. A further increase in the energy of ball lightning particles will occur by means of a transverse electromagnetic wave. There are no other possible mechanisms to explain the increase in proton energy during the ball lightning contact with a solid-state target.

\section{Theoretical Justification of the Super-Passability Phenomenon of the Ball Lightning}

A phenomenon analogous to the extensive air showers in the Earth's atmosphere occurs when the ball lightning interacts with a dense matter. As you know, a cascade process occurs when primary particles with high energy (protons) interact with a dense matter. As a result of the cascade process, primary particles decay into elementary particles [28]. The cascade process at the interaction of the primary particles with air was carefully studied in [29] [30]. The cascade process at the interaction of ball lightning with the dense matter is occurring in a following way. Due to the conversion of energy when ball lightning enters a dense matter, the kinetic energy of protons becomes sufficient to generate pions and kaons. It is also impossible to exclude the possible influence of the fact that ball lightning is a completely spin-polarized object. When protons with energies exceeding $140 \mathrm{MeV}$ collide with the nuclei of dense matter atoms, two types of pions are formed: neutral $\pi^{0}$ and charged: $\pi^{+}, \pi^{-}$. The neutral pions $\pi^{0}$ are then decayed into two photons, which create electrons and positrons in Coulomb field of the nuclei. The bremsstrahlung of the electron-positron pair leads to the appearance of an electro-magnetic shower consisting of low-energy gamma-quantums, namely photons. By interacting with the nuclei, the charged 
pions generate new particle showers. A scheme of cascade process at the interaction the protons of the ball lightning with a dense matter is given in Figure 8. As known the decay of a negative pion $\pi^{-}$causes an appearance of a negative muon together with a muon antineutrino

$$
\pi^{-} \rightarrow \mu^{-}+\tilde{v}_{\mu} .
$$

The decay of a positive pion $\pi^{+}$creates a positive muon with a muon neutrino

$$
\pi^{+} \rightarrow \mu^{+}+v_{\mu} .
$$

The negative muon itself is decayed into an electron, muon neutrino and electron antineutrino

$$
\mu^{-} \rightarrow e^{-}+v_{\mu}+\tilde{v}_{e} .
$$

Similarly, due to decay of the positive muon, a positron, electron neutrino and muon antineutrino appear

$$
\mu^{+} \rightarrow e^{+}+v_{e}+\tilde{v}_{\mu} .
$$

Due to the fact, that charged muons have the same charge value as electron, and the mass which exceeds the electron mass approximately 207 times, one can propose the following hypothesis. Let's suppose that muons persist the same

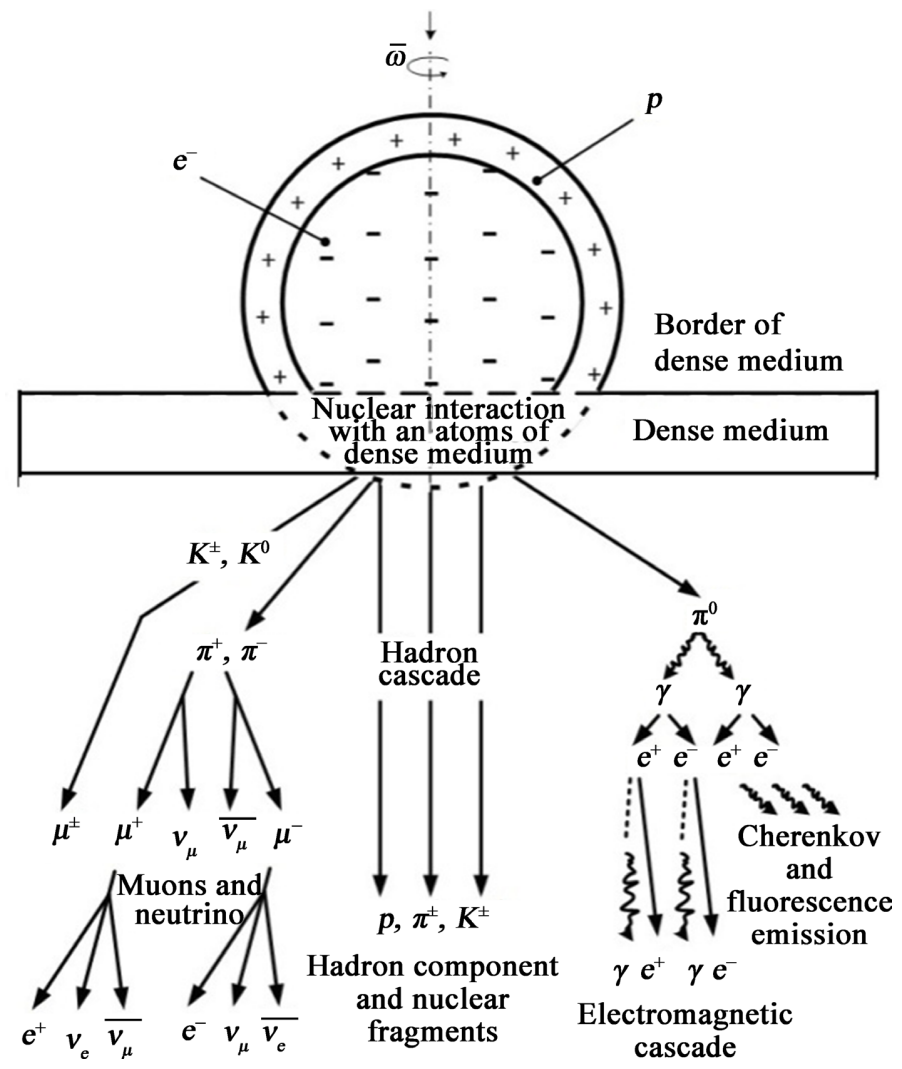

Figure 8. A schema of the cascade process at the interaction high-energy protons of the ball lightning with a dense matter of the solid-state sheet. Note: the magnetic field lines of the ball lightning are not shown in this sheme. 
movement direction, which the charged particles of the ball lightning have had before they reached the solid-state sheet. The muon decay is going to occur also above the surface of the dense matter. The interaction between the charged particles and neutral atoms of the air over the solid-state sheet will cause ionization of atoms. Simultaneously, the weak poloidal magnetic field of the entering ball lightning penetrates into the solid-state sheet. The induction of the penetrating magnetic field is changing in time. According to Maxwell equation changing in time magnetic field creates an electric field. The azimuthal (vortex) electric field will be created in the region over the solid-state sheet. The process of charges separation in the area above the solid-state sheet occurs due to the diffusion of charged particles across of the nonuniform magnetic field. The structural elements of the ball lightning receive a significant rotational moment due to the movement of charged particles in crossed electric and magnetic fields $\left(\boldsymbol{E}_{r} \times \boldsymbol{B}_{\theta}\right)$.

The core and the outer spherical shell of the ball lightning are created due to charge separation, which is caused by motion of charged particles in crossed longitudinal magnetic and azimuthal electric fields. The ball lightning elements with excess space charges rotating by azimuth (so-called outgoing ball lightning) appear as a result of the inequality of the charged particle fluxes above solid-state sheet. The formation of the outgoing ball lightning ends when the resultant of all forces in the equatorial plane becomes equal to zero. The conditions for a stable state of ball lightning are given in [10]. It should be noted that the cascade mechanism for explanation of the super-passability phenomenon of the ball lightning at first approximation has been proposed in [26]. A multitude of the above facts give sufficient grounds to believe that when the ball lightning enters a solid matter, a cascade process occurs, accompanied by the generation of muons and muon neutrino. This is the only way to explain the super-passability phenomenon of the ball lightning from the point of view of standard physics.

\section{On Possibility of Using the Ball Lightning for Muon-Catalyzed Fusion}

As you know, one of the relevant problems for humanity is the problem of an energy. Attempts to solve this problem for many years have been unsuccessful. There are many unsolved problems in thermonuclear fusion [31]. The main one is the problem of magnetic plasma confinement, which has no solution [32].

The problem of an energy based on the nuclear fusion reactions can be solved only if it performed in a low temperature medium with high frequency of collisions between components of mixture. There are sufficient grounds to believe that the concept of nuclear fusion based on muon catalysis [33] is the only correct approach for successfully solving the problem of nuclear fusion. This concept has been experimentally confirmed [34]. However, the cost of muons produced by giant accelerators is very high. This problem can be successfully solved 
if ultra-short duration laser [35] or ball lightning [36] is used for carry out muon-catalyzed fusion. It should be noted that facts of the water evaporation in large amounts when a small natural ball lightning hit the container have been confirmed in a number of observations. There are sufficient grounds to believe that the ball lightning is a natural reactor of nuclear fusion. The nuclear fusion reaction takes place when the ball lightning enters into the water due to the presence of deuterium in the water and appearance of the muons. The generation of the muons at interaction of the ball lightning with the target or the dense medium makes possible to use of their for nuclear fusion purposes. An interesting application is a periodic injection of the ball lightnings into the chamber of the reactor of nuclear fusion which was preliminary filled of dense low-temperature deuterium-tritium mixture [36]. The experiments were started in the laboratory on ball lightning interaction with deuterium-containing medium. As know, the optimal medium for the fusion reaction is a mixture of deuterium with tritium. The water and the water vapor were chosen by the authors because of the lack of possibility to work with a mixture of deuterium and tritium. The experiments were stopped due to unreasonable restrictions from officials in connection with the pandemic and the problem due to funding.

\section{Conclusion}

The experiments on the study of the ball lightning passage through the solidstate materials were carried out. The theoretical model, which allows to give an explanation for the phenomenon of anomalous passage of the ball lightning through the sheets from metal, was elaborated on the basis of obtained experimental data. The nature of phenomenon concludes in the fact that when the ball lightning enters into a dense medium occur a process of energy conversion of own magnetic field into the kinetic energy of charged particles. The collisions of high-energy protons of the ball lightning with the nuclei of the atoms of the dense medium lead to the generation of pions. The decay of the pions creates muons, neutrino, antineutrino or photons. In the experiments, the black ball lightning was also detected when a normal ball lightning passes through very thick solid-state sheets. The article also notes that there is some analogy between the ball lightning entering dense matter and the pulsar, which periodically creates fluxes of particles due to changes in their magnetosphere. The phenomenon of the generation of muons and neutrino at the entrance of the ball lightning into a dense medium opens up new perspectives in many areas of research and applications and will affect the possibility of solving the energy problem on the basis of muon-catalyzed fusion. It should be noted that methods for producing the ball lightning have been developed and tested many times on the created experimental installations of the "Prometheus" type. The results obtained in this work make a contribution to knowledge about the fundamental properties of matter in Nature. In the experiments, ball lightning with a maximum diameter of 1 meter was also obtained [37]. 


\section{Acknowledgements}

The authors are grateful to Dr. V.S. Netchitailo for helpful discussions and comments.

\section{Funding}

This research did not receive specific grants or financial support from funding agencies in the state, public, commercial or not-for-profit sectors and therefore, this paper cannot be subject to any sanctions or restrictions.

\section{Conflicts of Interest}

The authors declare no conflicts of interest regarding the publication of this paper.

\section{References}

[1] Arago, F. (1837) Notices Scientifiques: Sur le Tonnerre. Bachelier, Paris.

[2] Kapitsa, P.L. (1955) The Nature of Ball Lightning. In: Ritchie, D.J., Ed., Ball Lightning. A Collection of Soviet Research in English Translation, Consultants Bureau, New York, 11-16.

[3] Singer, S. (1971) The Nature of Ball Lightning. Springer, Boston. https://doi.org/10.1007/978-1-4684-1866-8

[4] Netchitailo, V.S. (2019) High-Energy Atmospheric Physics: Ball Lightning. Journal of High Energy Physics, Gravitation and Cosmology, 5, 360-374. https://doi.org/10.4236/jhepgc.2019.52020

[5] Nikitin, A.I., Bychkov, V.L., Nikitina, T.F. and Velichko, A.M. (2014) High-Energy Ball Lightning Observations. IEEE Transactions on Plasma Science, 42, 3906-3911. https://doi.org/10.1109/TPS.2014.2359235

[6] Schedrin, A.I. (2018) Relativistic Ball Lightning Model. Goryachaya Liniya-Telecom, Moscow.

[7] Lowke, J.J. (1996) A Theory of Ball Lightning as an Electric Discharge. Journal Physics D: Applied Physics, 29, 1237-1244. https://doi.org/10.1088/0022-3727/29/5/018

[8] Koloc, P.M. (1989) PLASMAK ${ }^{\mathrm{TM}}$ Star Power for Energy Intensive Space Applications. Fusion Science and Technology, 15, 1136-1141. https://doi.org/10.13182/FST89-A39846

[9] Oreshko, A.G. (2006) Generation of Laboratory Ball Lightning. Journal of Physics. Conference Series, 44, 127-132. https://doi.org/10.1088/1742-6596/44/1/016

[10] Oreshko, A.G. (2015) An Investigation of the Generation and Properties of Laboratory-Produced Ball Lightning. Journal of Plasma Physics, 81, Article ID: 905810321. https://doi.org/10.1017/S0022377815000197

[11] Kozyrev, N.A. (1991) Selected Works. LGU, Leningrad.

[12] Le Sage, G.L. (1818) Physique Mécanique des Georges-Louis Le Sage. In: Prévost, P., Ed., Deux Traites de Physique Mécanique, J.J. Paschoud Books, Geneva \& Paris, 1-186.

[13] Oreshko, A.G. (2011) Ball Lightning Generation Research. International Journal Unconventional Electromagnetics and Plasmas, 3, 77-81.

[14] Oreshko, A.G. (2010) Ball Lightning Investigations on "Prometheus-2". Proceedings 
of the 37 th European Physical Society Conference on Plasma Physics, Dublin, 21-25 June 2010. http://ocs.ciemat.es/EPS2010PAP/PDF/P5.401.pdf

[15] Lowke, J.J., Smith, D., Nelson, K.E., Crompton, R.W. and Murphy, A.B. (2012) Birth of Ball Lightning. Journal of Geophysical Research: Atmospheres, 117, Article ID: D19107. https://doi.org/10.1029/2012JD017921

[16] Bychkov, V.L., Nikitin, A.I., Ivanenko, I.P., Nikitina, T.F., Velichko, A.M. and Nosikov, I.A. (2016) Ball Lightning Passage through a Glass without Breaking It. Journal of Atmospheric and Solar-Terrestrial Physics, 150-151, 69-76. https://doi.org/10.1134/S0026893316010222

[17] Oreshko, A.G., Oreshko, A.A. and Mavlyudov, T.B. (2019) Proton-Electron Model of Ball Lightning Structure. Journal of Atmospheric and Solar-Terrestrial Physics, 182, 194-199. https://doi.org/10.1016/j.jastp.2018.11.017

[18] Oreshko, A.G. (2012) The Effect of Anomalous Passing of Ball Lightning through Absorbing Filters. Proceeding of the 39 th European Physical Society Conference and 16th International Congress on Plasma Physics, Stockholm, 2-6 July 2012. http://ocs.ciemat.es/epsicpp2012pap/pdf/P5.107.pdf

[19] Thornhill, W. (2006) The IEEE, Plasma Cosmology and Extreme Ball Lightning. https://www.holoscience.com/wp/the-ieee-plasma-cosmology-and-extreme-ball-lig htning/

[20] Robinson, A.P.L. and Sherlock, M. (2007) Magnetic Collimation of Fast Electrons Produced by Ultraintense Laser Irradiation by Structuring the Target Composition. Physics of Plasmas, 14, Article ID: 083105. https://doi.org/10.1063/1.2768317

[21] Chatterjee, G., Singh, P.K., Robinson, A.P.L., Blackman, D., Booth, N., Dance, R.J., Gizzi, L.A., Gray, R.J. and Green, J.S. (2017) Micron-Scale Mapping of Megagauss Magnetic Fields Using Optical Polarimetry to Probe Hot Electron Transport in Petawatt-Class Laser-Solid Interactions. Scientific Report, 7, Article No. 8347. https://doi.org/10.1038/s41598-017-08619-1

[22] Oreshko, A.G. (2009) Research of a Ball Lightning and Prospects of It's Use for Applied Purposes. Proceedings of the VI International Conference on Plasma Physics and Plasma Technology. Contributed Papers, Minsk, 1, 137-140.

[23] Rabinowitz, M. (2001) n-Dimensional Gravity: Little Black Holes, Dark Matter and Ball Lightning. International Journal of Theoretical Physics, 40, 875-901.

https://doi.org/10.1023/A:1004152729335

[24] Oreshko, A.G. (2001) Generation of Strong Fields in Plasma. Doklady Physics, 46, 9-11. https://doi.org/10.1134/1.1348578

[25] Oreshko, A.G. and Mavlyudov, T.B. (2011) The Effect of Hot Plasmoids Generation in High-Current Vortex Discharge under Atmospheric Conditions. IEEE Transactions on Plasma Science, 39, 2124-2125.

https://doi.org/10.1109/TPS.2011.2165298

[26] Oreshko, A.G. and Oreshko, A.A. (2016) Investigation Ball Lightning Penetration through Absorbing Filters. Proceedings of the 43rd European Physical Society Conference on Plasma Physics, Leuven, 4-8 July 2016. http://ocs.ciemat.es/EPS2016PAP/pdf/P2.110.pdf

[27] Auger, P., Ehrenfest, P., Maze, R., Daudin, J. and Fréon, R.A. (1939) Extensive Cos mic-Ray Showers. Reviews of Modern Physics, 11, 288-291.

https://doi.org/10.1103/RevModPhys.11.288

[28] Grupen, C. (2005) Astroparticle Physics. Springer, Berlin.

[29] Heber, B., Kota, J. and von Steiger, R. (2014) Cosmic Rays in the Heliosphere: Tem- 
poral and Spatial Variations. Springer, New York.

[30] Stanev, T. (2010) High Energy Cosmic Rays. 2nd Edition, Springer, New York.

[31] Linhart, G.J. (2009) Quo Vadis Fusion? Nukleonika, 54, 305-310. http://www.nukleonika.pl/www/back/full/vol54_2009/v54n4p305f.pdf

[32] Oreshko, A.G. (2012) The Electrical Domains and Anomalous Phenomenons in Plasma. Proceeding of the 39th European Physical Society Conference and 16 th International Congress on Plasma Physics, Stockholm, 2-6 July 2012. http://ocs.ciemat.es/epsicpp2012pap/pdf/P4.181.pdf

[33] Frank, F.C. (1947) Hypothetical Alternative Energy Sources for the "Second Meson" Events. Nature, 160, 525-527. https://doi.org/10.1038/160525a0

[34] Alvarez, L.W., Bradner, H., Crawford, F.S., Crawford, J.A., Falk-Vairant, P., Good, M.L, Gow, J.D., Rosenfeld, A.H., Solmitz, F.M., Stevenson, L., Ticho, H.K. and Tripp, R.D. (1957) Catalysis of Nuclear Reactions by $\mu$ Mesons. Physical Review, 105, 1127-1128. https://doi.org/10.1103/PhysRev.105.1127

[35] Andersson, P.U. and Holmlid, L. (2012) Fusion Generated Fast Particles by Laser Impact on Ultra-Dense Deuterium: Rapid Variation with Laser Intensity. Journal of Fusion Energy, 31, 249-256. https://doi.org/10.1007/s10894-011-9468-2

[36] Oreshko, A.G., Oreshko, A.A. and Mavlyudov, T.B. (2018) On Possibility of Creating a Muon-Catalytic Reactor Based on Periodic Injection of Ball Lightnings in a Chamber with D-T Mixture. Proceedings of the 45 th European Physical Society Conference on Plasma Physics, Prague, 2-6 July 2018.

http://ocs.ciemat.es/EPS2018PAP/pdf/P1.2031.pdf

[37] Oreshko, A.G. (2021) On Physical Investigation of Ball Lightnings. Journal of Plasma Physics. 